\title{
The Importance of Clinicians Reviewing CT Scans in Suspected Lacrimal Gland Disease Causing Eyelid Swelling, Even if Radiologists Previously Interpreted them as Normal
}

\author{
George Voyatzis ${ }^{1}$, Lekha Chandrasekharan ${ }^{2}$, Ian Francis ${ }^{2}$ and Raman Malhotra ${ }^{*}, 1$ \\ ${ }^{I}$ Corneo Plastic Unit, Queen Victoria Hospital NHS Foundation Trust, Holtye Road, East Grinstead, West Sussex RH19 \\ $3 D Z, U K$ \\ ${ }^{2}$ Radiology Department, Queen Victoria Hospital NHS Foundation Trust, Holtye Road, East Grinstead, West Sussex \\ RH19 3DZ, UK
}

\begin{abstract}
Purpose: To highlight the importance of deliberate evaluation of the lacrimal gland during routine orbital imaging by both radiologists and clinicians, which may avoid delays in diagnosis of eyelid swelling related to lacrimal gland disease.

Methods: We present four cases referred to a tertiary ophthalmic plastic and orbital centre for assessment of chronic upper eyelid swelling of unknown aetiology. All four cases had been investigated with orbital CT imaging, reported to be normal by consultant radiologists.

Results: Careful review of existing imaging provided valuable information concerning the lacrimal gland and helped identify a pathological cause in all four cases.

Conclusions: Lacrimal gland disease can be missed radiologically without evaluation of coronal as well as standard axial orbital imaging. Clear communication with the radiologist as well as personally reviewing scans can avoid such pitfalls.
\end{abstract}

Keywords: Lacrimal gland, orbital imaging, eyelid swelling.

Eyelid swelling due to lacrimal gland disease is usually confirmed based on an obvious abnormality on orbital imaging.

We present four cases referred to a tertiary ophthalmic plastic and orbital centre for assessment of chronic upper eyelid swelling of unknown aetiology. All four had orbital CT scans, reported to be normal by four different consultant radiologists. Careful review of the existing imaging in all four cases identified obvious lacrimal gland pathology that helped establish a diagnosis.

This series emphasises the importance of evaluating coronal and axial CT views for suspected lacrimal gland disease.

\section{CASE REPORTS}

\section{Case 1}

A 67-year-old female patient was referred, suspected to have thyroid eye disease presumably secondary to Interferon treatment for Hepatitis C. She had bilateral eyelid swelling. (Fig. 1) Her orbit CT scan report mentioned mild enlargement of her extraocular muscles.

On examination the lacrimal gland was not clinically enlarged however she had an s-shaped lateral ptosis and she

\footnotetext{
*Address correspondence to this author at the Corneo Plastic Unit, Queen Victoria Hospital NHS Foundation Trust, Holtye Road, East Grinstead, West Sussex RH19 3DZ, UK; Tel: 01342 4144560; Fax: 1342330363;

E-mail: raman.malhotra@qvh.nhs.uk
}

was aware of tenderness in this area on both sides. The existing CT scans clearly showed bilateral enlarged lacrimal glands with diffuse ill-defined infiltrative edges on coronal views. A lacrimal gland biopsy and a raised serum ACE confirmed the diagnosis of orbital sarcoidosis.

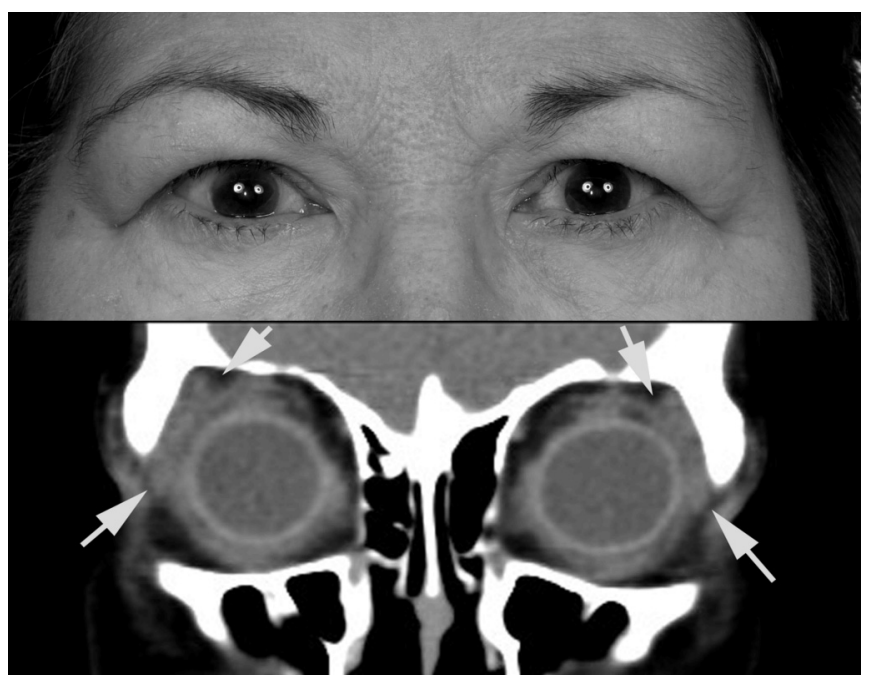

Fig. (1). Patient presenting with bilateral eyelid swelling. The CT scans clearly showed bilateral enlarged lacrimal glands with diffuse ill-defined infiltrative edges on coronal views.

\section{Case 2}

A 58-year-old female was referred with a 10-year history of intermittent swelling of her eyelids with no obvious cause. 
Her medication included methotrexate for rheumatoid arthritis. On examination, she had a slight s-shaped ptosis of her upper eyelids and bilateral xanthelasma. (Fig. 2) The CT scans report mentioned only subtle thickening of her right and left inferior recti. Review of the CT scans showed diffuse solid infiltration around both lacrimal glands. A lacrimal gland biopsy confirmed a diagnosis of orbital xanthogranuloma.

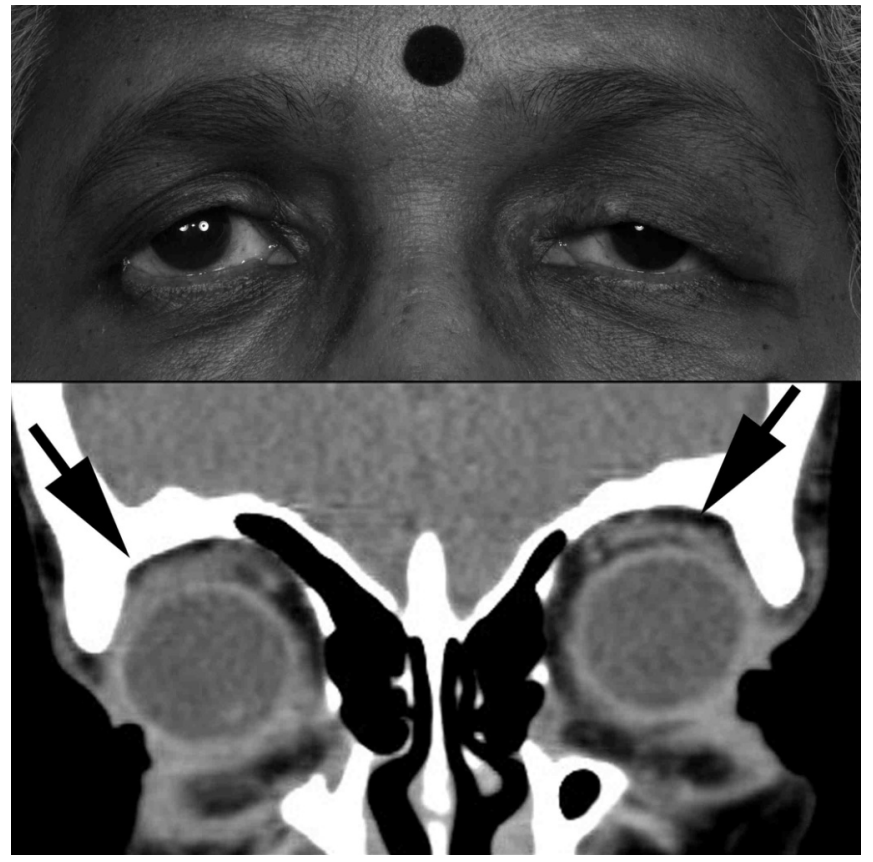

Fig. (2). Patient presenting with slight s-shaped ptosis of her upper eyelids and bilateral xanthelasma. CT scans show diffuse solid infiltration around both lacrimal glands.

\section{Case 3}

A 45-year-old female referred with a 3-week history of unilateral eyelid swelling (Fig. 3). Her orbits CT scan was reported as normal. She was then presumed to have an allergy related eyelid swelling and observed. She was subsequently referred due to persistence of inflammation. Careful re-evaluation of coronal CT orbital imaging revealed a clearly enlarged lacrimal gland with ill-defined margins consistent with dacryoadenitis. A lacrimal gland biopsy confirmed dacryoadenitis as part of idiopathic orbital inflammation. Her symptoms and signs resolved following a 6-week regime of oral steroids.

\section{Case 4}

An 80-year-old male patient was referred with a twomonth history of a left painless upper eyelid swelling. The orbit CT scan was reported as normal. On examination, the main finding was a descended left lacrimal gland. Review of the CT imaging showed proptosis of the left lacrimal gland with a prominent fat pocket posteriorly in comparison to the right side. Surgery, with repositioning of the lacrimal gland and septal plication through a transcutaneous approach was undertaken.

\section{Comment}

This case series emphasises the importance of careful evaluation by the clinicians of the lacrimal gland imaging by personally reviewing both axial and coronal views of the CT scans even if radiologists interpreted them as normal.

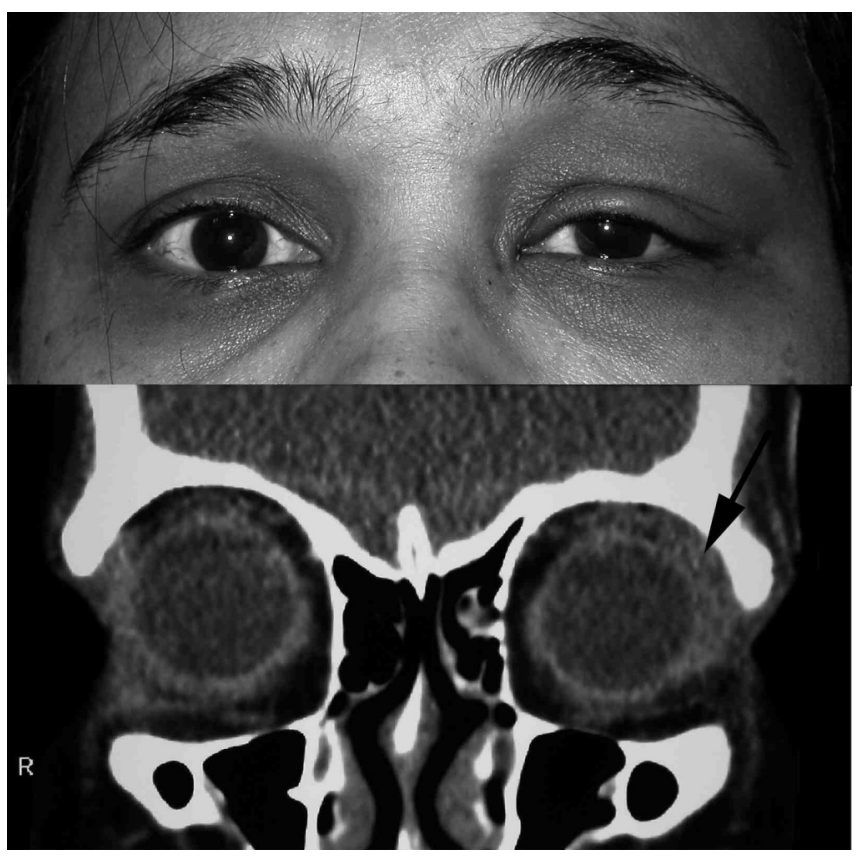

Fig. (3). Patient presenting with unilateral eyelid swelling. The coronal CT orbital imaging revealed a clearly enlarged lacrimal gland with ill-defined margins.

In all four cases, careful review by the oculoplastic clinicians of the reported as normal existing scans, in particular the coronal views, helped identify previously missed obvious lacrimal gland pathology.

Proper evaluation of lacrimal gland imaging can identify underlying pathology, avoid unnecessary referrals and speed up the diagnosis and treatment of the disease [1-3].

The normal lacrimal gland is located in the superior tarsal aspect of the orbit, is roughly the same size and shape as an almond, extraconal in position and extends deep into the orbital septum. It consists of the anterior palpebral and deeper orbital lobes. The anterior palpebral lobe is approximately one-third to one- half the size of the orbital lobe. Most of the lacrimal gland tumours arise in the orbital lobe. The clinician should evaluate both axial and coronal views of both bone and soft tissue windows. As a first step the definition of the edges of the gland and any possible enlargement unilateral or bilateral must be determined. If a mass is present, its relationship to other structures and the presence of infiltrative disease must be assessed. The doctor needs to evaluate possible posterior extension into the orbital soft tissues and the presence or absence of bone deformity or destruction. It is also important to identify the presence of calcification and observe for the patterns of contrast enhancement. By following these basic steps, the referring ophthalmologist will be able to identify most of lacrimal gland pathology by personally reviewing the CT scans.

Lacrimal gland related causes of eyelid swelling can be missed without recognition of typical features and appropriate evaluation of coronal in conjunction with standard axial orbital imaging [4]. The clinician suspecting lacrimal gland disease and requesting orbital imaging should always communicate with the radiologist requesting both 
axial and coronal $\mathrm{CT}$ views and also consider reviewing the scans themselves and not rely solely on the written report.

\section{REFERENCES}

[1] Jung WS, Ahn KJ, Park MR, et al. The Radiological Spectrum of Orbital Pathologies that Involve the Lacrimal Gland and the Lacrimal Fossa. Korean J Radiol 2007; 8(4): 336-42.
[2] Gorospe L, Royo A, Berrocal T, et al. Imaging of orbital disorders in paediatric patients. Eur Radiol 2003; 13: 2012-26.

[3] Maya MM, Heier LA. Orbital CT. Current use in the MR era. Neuroimaging Clin N Am 1998; 8: 175-88.

[4] Warner MA, Weber AL, Jacobiec FA. Benign and malignant tumors of the orbital cavity including the lacrimal gland. Neuroimaging Clin N Am 1996; 6: 123-42.

(C) Voyatzis et al.; Licensee Bentham Open.

This is an open access article licensed under the terms of the Creative Commons Attribution Non-Commercial License (http: //creativecommons.org/licenses/by$\mathrm{nc} / 3.0 /$ ) which permits unrestricted, non-commercial use, distribution and reproduction in any medium, provided the work is properly cited. 